\title{
Spherical topological-insulator nanoparticles: Quantum size effects and optical transitions
}

\author{
L. Gioia, ${ }^{1,2}$ M. G. Christie, ${ }^{3}$ U. Zülicke $,{ }^{3,4}, *$ M. Governale,${ }^{3}$ and A. J. Sneyd ${ }^{3}$ \\ ${ }^{1}$ Perimeter Institute, 31 Caroline Street North, Waterloo, Ontario, Canada N2L 2 Y5 \\ ${ }^{2}$ Department of Physics and Astronomy, University of Waterloo, Waterloo, Ontario, Canada N2L 3G1 \\ ${ }^{3}$ School of Chemical and Physical Sciences and MacDiarmid Institute for Advanced Materials and Nanotechnology, \\ Victoria University of Wellington, PO Box 600, Wellington 6140, New Zealand \\ ${ }^{4}$ Department of Physics, University of Basel, Klingelbergstrasse 82, CH-4056 Basel, Switzerland
}

(Dated: November 4, 2019)

\begin{abstract}
We have investigated the interplay between band inversion and size quantization in spherically shaped nanoparticles made from topological-insulator (TI) materials. A general theoretical framework is developed based on a versatile continuum-model description of the TI bulk band structure and the assumption of a hard-wall mass confinement. Analytical results are obtained for the wave functions of single-electron energy eigenstates and the matrix elements for optical transitions between them. As expected from spherical symmetry, quantized levels in TI nanoparticles can be labeled by quantum numbers $j$ and $m=-j,-j+1, \ldots, j$ for total angular momentum and its projection on an arbitrary axis. The fact that TIs are narrow-gap materials, where the charge-carrier dynamics is described by a type of two-flavor Dirac model, requires $j$ to assume half-integer values and also causes a doubling of energy-level degeneracy where two different classes of states are distinguished by being parity eigenstates with eigenvalues $(-1)^{j \mp 1 / 2}$. The existence of energy eigenstates having the same $j$ but opposite parity enables optical transitions where $j$ is conserved, in addition to those adhering to the familiar selection rule where $j$ changes by \pm 1 . All optical transitions satisfy the usual selection rule $\Delta m=0, \pm 1$. We treat intra- and inter-band optical transitions on the same footing and establish ways for observing unusual quantum-size effects in TI nanoparticles, including oscillatory dependences of the band gap and of transition amplitudes on the nanoparticle radius. Our theory also provides a unified perspective on multi-band models for charge carriers in semiconductors and Dirac fermions from elementary-particle physics.
\end{abstract}

\section{INTRODUCTION AND SYNOPSIS OF MAIN RESULTS}

Synthesis and experimental investigation of semiconductor nanocrystals have been pursued with great effort to elucidate how electronic and optical properties of such low-dimensional systems are affected by size-quantization effects $[1,2]$. Recent advances in the fabrication of nanoparticles made from topological-insulator (TI) materials [3-9] have created the opportunity to explore the interplay of topological properties and quantum confinement [10]. In particular, TI materials are known to host surface states that are robust to perturbations and have energies within the bulk band gap [11-13]. The presence of these surface states is envisioned to enable intriguing applications for TI-based optoelectronics [14], spintronics $[15,16]$, and thermoelectrics [17]. As device fabrication often involves nanostructuring of the TI material, it is necessary to understand the evolution of surface-state properties as the TI nanomaterial's size is reduced [1823]. Our theoretical description for the electronic and optical properties of spherical TI nanoparticles sheds new light on the importance and implications of quantum-size effects in these, and related, nanostructures.

Previous theoretical studies of TI nanoparticles have used atomistic electronic-structure calculations [24], ap-

*uli.zuelicke@vuw.ac.nz proximate descriptions for the surface states [25, 26], discretized versions of effective $\mathbf{k} \cdot \mathbf{p}$ models [25, 27-29], and numerical exact diagonalization $[30,31]$ to discuss excitonic properties [24], effects of interactions [30] and disorder [28, 31], as well as the emergence of unusual electromagnetic properties [27, 29]. Related works have considered idealized Dirac models in curved spaces [32$34]$ or subject to spherically symmetric quantum confinement $[35,36]$. Here we obtain the exact spectrum of quantised energies and associated quantum states for nanoparticle confinement of charge carriers described by a spherically symmetric version of the low-energy continuum-model Hamiltonian for bulk TIs [37-39],

$$
\begin{aligned}
H= & \left(\frac{\Delta_{0}}{2}+\frac{\gamma}{k_{\Delta}} \mathbf{k}^{2}\right) \tau_{z} \otimes \sigma_{0} \\
& +\gamma\left(k_{z} \tau_{x} \otimes \sigma_{z}+k_{-} \tau_{x} \otimes \sigma_{+}+k_{+} \tau_{x} \otimes \sigma_{-}\right),
\end{aligned}
$$

comprising both pseudo-spin $(\tau)$ and real-spin $(\sigma)$ degrees of freedom [40]. In Eq. (1), $\mathbf{k} \equiv\left(k_{x}, k_{y}, k_{z}\right)=-i \boldsymbol{\nabla}$ is the operator for the single-electron wave vector, and $k_{ \pm}=k_{x} \pm i k_{y}$. Further details of the formalism are discussed in Sec. II, and Table I provides the parameter values applicable to a number of currently available TI materials. We use the remainder of this Section to present a selection of key results.

The nanoparticle confinement leads to the emergence of quantized energies $E_{j}^{(n)}$ for single-electron states labeled by the half-integer total-angular-momentum quantum number $j$ and a radial quantum number $n$. For 

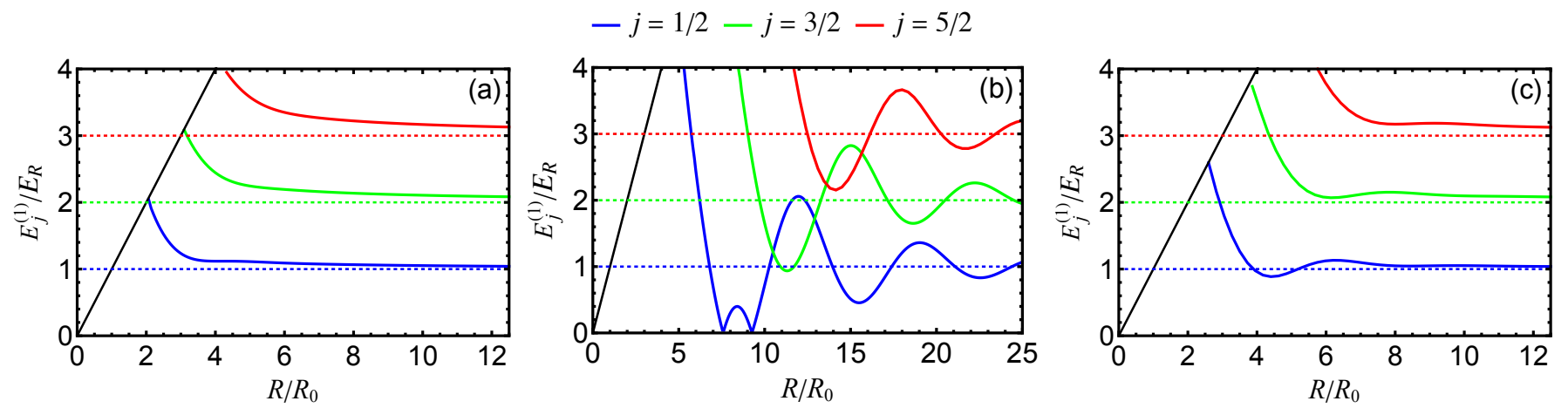

FIG. 1. Energies $E_{j}^{(1)}$ of quantized electron levels created from topologically protected surface states by a spherical hard-wall mass-confinement potential with radius $R$. The solid blue (green, red) curve in each panel corresponds to the state with totalangular-momentum quantum number $j=1 / 2(j=3 / 2, j=5 / 2)$. Here $E_{R}=\gamma / R$ is the size-quantisation energy scale and $R_{0}=2 \gamma /\left|\Delta_{0}\right|$ the bulk Compton length. Results shown in panel (a), (b) and (c) were obtained for fixed $\Delta_{0} /\left(\gamma k_{\Delta}\right)=-1.2$, -9.5 and -2.3 , respectively, which are values representative of $\mathrm{Bi}_{2} \mathrm{Se}_{3}, \mathrm{Bi}_{2} \mathrm{Te}_{3}$ and $\mathrm{Sb}_{2} \mathrm{Te}_{3}$ (see Table I for these materials' band-structure parameters). The thin black line indicates the bulk-material conduction-band edge.

TABLE I. Values adopted for parameters in the spherically symmetric effective bulk continuum-model Hamiltonian (1) to represent currently available topological-insulator materials. The Compton length $R_{0} \equiv 2 \gamma /\left|\Delta_{0}\right|$ for bulk charge carriers is indicative for the magnitude of the nanoparticle radius $R$ below which the band structure becomes topologically trivial.

\begin{tabular}{lcccc}
\hline \hline & $\gamma(\mathrm{eV} \AA)$ & $\Delta_{0}(\mathrm{eV})$ & $k_{\Delta}\left(\AA^{-1}\right)$ & $R_{0}(\mathrm{~nm})$ \\
\hline $\mathrm{Bi}_{2} \mathrm{Te}_{3}{ }^{\mathrm{a}}$ & 2.4 & -0.592 & 0.026 & 0.81 \\
$\mathrm{Sb}_{2} \mathrm{Te}_{3}{ }^{\mathrm{a}}$ & 2.4 & -0.364 & 0.066 & 1.3 \\
$\mathrm{Bi}_{2} \mathrm{Se}_{3}{ }^{\mathrm{a}}$ & 2.2 & -0.338 & 0.13 & 1.3 \\
$\mathrm{HgTe}^{\mathrm{b}}$ & 8.4 & -0.303 & 0.09 & 5.5 \\
$\mathrm{~Pb}_{0.81} \mathrm{Sn}_{0.19} \mathrm{Se}^{\mathrm{c}}$ & 3.2 & -0.025 & 0.20 & 26 \\
$\mathrm{~Pb}_{0.54} \mathrm{Sn}_{0.46} \mathrm{Te}^{\mathrm{d}}$ & 4.8 & -0.030 & $\gtrsim 0.25$ & 32 \\
\hline \hline
\end{tabular}

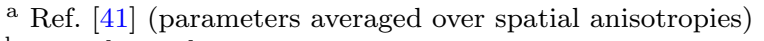

b Refs. $[42,43]$

${ }^{c}$ Ref. [44]

d Ref. [45]

large-enough nanoparticle radius $R$, the lowest positiveenergy levels $E_{j}^{(1)}$ are below the bulk conduction-band edge $\Delta_{0} / 2$. These remnants of the topologically protected surface states of the bulk system are pushed to higher energies as $R$ is reduced and, one-by-one starting from the largest $j$, disappear from the subgap energy range. This is illustrated for the three lowest positive- energy levels in Fig. 1, where we adopted the TI-material Compton length $R_{0} \equiv 2 \gamma /\left|\Delta_{0}\right|$ and the size-quantization energy scale $E_{R} \equiv \gamma / R$ as natural units. The values for $\Delta_{0} /\left(\gamma k_{\Delta}\right)$ used in the calculation of results shown in panels (a), (b) and (c) are derived from band-structure parameters given in Table I for $\mathrm{Bi}_{2} \mathrm{Se}_{3}, \mathrm{Bi}_{2} \mathrm{Te}_{3}$ and $\mathrm{Sb}_{2} \mathrm{Te}_{3}$, respectively. Clear deviations from the previously determined $[25,36]$ asymptotic large- $R$ behavior $E_{|\lambda|}^{(1)} \sim$ $\left(j+\frac{1}{2}\right) E_{R}$ occur over a wider range of $R / R_{0}$ for larger $j$ and increasing $\left|\Delta_{0}\right| /\left(\gamma k_{\Delta}\right) . \mathrm{Bi}_{2} \mathrm{Te}_{3}$ represents a case with strong deviations in the form of large oscillations and a steep rise in bound-state energy at the smallest values of $R / R_{0}$. The $\mathrm{Bi}_{2} \mathrm{Te}_{3}$ nanoparticle also exhibits gaplessness at two values of $R / R_{0}$, and there is a finite range of radii where the $j=3 / 2$ level is the lowest-energy state and thus defines the TI-nanoparticle band gap. Similar subband inversions occur between higher- $j$ levels. The lowest energy level is pushed above the bulk-conductionband edge for $R<R_{\mathrm{c}}=2.0 R_{0}\left(2.6 R_{0}, 5.0 R_{0}\right)$ in the system representing a $\mathrm{Bi}_{2} \mathrm{Se}_{3}\left(\mathrm{Sb}_{2} \mathrm{Te}_{3}, \mathrm{Bi}_{2} \mathrm{Te}_{3}\right)$ nanoparticle, marking the transition to a nontopological band structure. Thus although $R_{0}$ sets the overall scale for the critical radius below which the TI-nanoparticle spectrum becomes ordinary, the actual value of $R_{\mathrm{c}}$ is typically several times larger.

The wave functions in position ( $\mathbf{r}$ ) space for TInanoparticle-confined electrons can be written in the universal form

$$
\Psi_{j m+}^{(n)}(\mathbf{r})=\frac{C_{j+}^{(n)}}{2}\left(\begin{array}{c}
\sqrt{\frac{j+m}{j}} Y_{j-\frac{1}{2}}^{m-\frac{1}{2}}(\theta, \varphi) \phi_{j+\uparrow}^{(n)}(r) \\
\sqrt{\frac{j+1-m}{j+1}} Y_{j+\frac{1}{2}}^{m-\frac{1}{2}}(\theta, \varphi) \phi_{j-\uparrow}^{(n)}(r) \\
\sqrt{\frac{j-m}{j}} Y_{j-\frac{1}{2}}^{m+\frac{1}{2}}(\theta, \varphi) \phi_{j+\uparrow}^{(n)}(r) \\
-\sqrt{\frac{j+1+m}{j+1}} Y_{j+\frac{1}{2}}^{m+\frac{1}{2}}(\theta, \varphi) \phi_{j-\uparrow}^{(n)}(r)
\end{array}\right), \Psi_{j m-}^{(n)}(\mathbf{r})=\frac{C_{j-}^{(n)}}{2}\left(\begin{array}{c}
\sqrt{\frac{j+1-m}{j+1}} Y_{j+\frac{1}{2}}^{m-\frac{1}{2}}(\theta, \varphi) \phi_{j+\uparrow}^{(n)}(r) \\
\sqrt{\frac{j+m}{j}} Y_{j-\frac{1}{2}}^{m-\frac{1}{2}}(\theta, \varphi) \phi_{j-\uparrow}^{(n)}(r) \\
-\sqrt{\frac{j+1+m}{j+1}} Y_{j+\frac{1}{2}}^{m+\frac{1}{2}}(\theta, \varphi) \phi_{j+\uparrow}^{(n)}(r) \\
\sqrt{\frac{j-m}{j}} Y_{j-\frac{1}{2}}^{m+\frac{1}{2}}(\theta, \varphi) \phi_{j-\uparrow}^{(n)}(r)
\end{array}\right)
$$


associated with the ordered set of basis states [37-39]

$$
\begin{aligned}
& \left|P 1_{-}^{+}, 1 / 2\right\rangle \equiv|+\uparrow\rangle,-i\left|P 2_{+}^{-}, 1 / 2\right\rangle \equiv|-\uparrow\rangle, \\
& \quad\left|P 1_{-}^{+},-1 / 2\right\rangle \equiv|+\downarrow\rangle, i\left|P 2_{z}^{-},-1 / 2\right\rangle \equiv|-\downarrow\rangle .
\end{aligned}
$$

Here $Y_{j \pm \frac{1}{2}}^{m \pm \frac{1}{2}}(\theta, \varphi)$ are the familiar spherical harmonics as defined in Ref. [46]. The physically unimportant phase factors $C_{j \kappa}^{(n)}$ are given below [see Eq. (20)]; they were chosen to make contact with mathematical formalisms used to describe massless Dirac fermions confined to move on the surface of the unit sphere [32]. The functions $\phi_{j \tau \sigma}^{(n)}(r)$ are determined by solving a radial Schrödinger equation as explained in greater detail in Sec. II. Noting that the spherical harmonics $Y_{|\lambda|}^{\mu}$ acquire a factor $(-1)^{|\lambda|}$ under the parity transformation [46], and that the basis states $|+\sigma\rangle(|-\sigma\rangle)$ are even (odd) under parity [37-39], the wave functions $\Psi_{j m \pm}^{(n)}(\mathbf{r})$ are found to be parity eigenstates with eigenvalue $(-1)^{j \mp \frac{1}{2}}$. The angular part of the wave functions from Eq. (2) has a structure analogous to that exhibited by localized states in narrow-gap semiconductors $[47,48]$ and spherically symmetric solutions of the Dirac equation [49].

The explicit form of the wave functions (2) enables straightforward calculation of the amplitude for optical transitions between quantized electron states in a TI nanoparticle. In Sec. III, we derive the general expression

$$
\mathbf{d}=e \mathbf{r} \tau_{0} \otimes \sigma_{0}+\frac{e R_{0}}{2} \tau_{y} \otimes \boldsymbol{\sigma}
$$

for the optical-dipole operator acting in 4-dimensional envelope-function space of combined pseudo-spin $(\tau)$ and real-spin $(\sigma)$ degrees of freedom [40] describing electrons in the TI material [50]. The optical matrix elements

$$
\mathbf{d}_{n j m \kappa}^{n^{\prime} j^{\prime} m^{\prime} \kappa^{\prime}} \equiv \int d^{3} r\left[\Psi_{j^{\prime} m^{\prime} \kappa^{\prime}}^{\left(n^{\prime}\right)}(\mathbf{r})\right]^{\dagger} \mathbf{d} \Psi_{j m \kappa}^{(n)}(\mathbf{r}),
$$

for the transition from an initial state $\Psi_{j m \kappa}^{(n)}$ to a final state $\Psi_{j^{\prime} m^{\prime} \kappa^{\prime}}^{\left(n^{\prime}\right)}$ are explicitly found as

$$
\begin{array}{r}
\left(d_{x} \pm i d_{y}\right)_{n j m \kappa}^{n^{\prime} j^{\prime} m^{\prime} \kappa^{\prime}}=e \delta_{m^{\prime}, m \pm 1}\left\{\delta_{\kappa^{\prime}, \kappa} \sum_{\xi= \pm 1} \frac{\sqrt{\left[j+\frac{1}{2}+\xi\left(\frac{1}{2} \pm m\right)\right]\left[j+\frac{1}{2}+\xi\left(\frac{3}{2} \pm m\right)\right]}}{2 j+1+\xi}\left(\mp \xi \Re_{n j}^{n^{\prime} j^{\prime}} \pm i \kappa \frac{R_{0}}{2} \delta_{n j,-\kappa \xi}^{n^{\prime} j^{\prime}, \kappa \xi}\right) \delta_{j^{\prime}, j+\xi}\right. \\
\left.+\delta_{\kappa^{\prime},-\kappa} \frac{\sqrt{(j \mp m)(j+1 \pm m)}}{2 j(j+1)}\left(\Re_{n j}^{n^{\prime} j^{\prime}}+i \kappa \frac{R_{0}}{2}\left[j \delta_{n j,-\kappa}^{n^{\prime} j^{\prime}, \kappa}+(j+1) \delta_{n j, \kappa}^{n^{\prime} j^{\prime},-\kappa}\right]\right) \delta_{j^{\prime}, j}\right\}\left[C_{j^{\prime} \kappa^{\prime}}^{\left(n^{\prime}\right)}\right]^{*} C_{j \kappa}^{(n)}, \quad(6 \mathrm{a}) \\
\left(d_{z}\right)_{n j m \kappa}^{n^{\prime} j^{\prime} m^{\prime} \kappa^{\prime}}=e \delta_{m^{\prime}, m}\left\{\delta_{\kappa^{\prime}, \kappa} \sum_{\xi= \pm 1} \frac{\sqrt{\left[j+(1+\xi) \frac{1}{2}+m\right]\left[j+(1+\xi) \frac{1}{2}-m\right]}}{2 j+1+\xi}\left(\mathscr{R}_{n j}^{n^{\prime} j^{\prime}}-i \kappa \xi \frac{R_{0}}{2} \delta_{n j,-\kappa \xi}^{n^{\prime} j^{\prime}, \kappa \xi}\right) \delta_{j^{\prime}, j+\xi}\right. \\
\left.+\delta_{\kappa^{\prime},-\kappa} \frac{m}{2 j(j+1)}\left[\mathscr{R}_{n j}^{n^{\prime} j^{\prime}}+i \kappa \frac{R_{0}}{2}\left(j \delta_{n j,-\kappa}^{n^{\prime} j^{\prime}, \kappa}+(j+1) \delta_{n j, \kappa}^{n^{\prime} j^{\prime},-\kappa}\right)\right] \delta_{j^{\prime}, j}\right\}\left[C_{j^{\prime} \kappa^{\prime}}^{\left(n^{\prime}\right)}\right]^{*} C_{j \kappa}^{(n)} . \quad(6 \mathrm{~b})
\end{array}
$$

For convenience, we have introduced the abbreviation

$$
\mathscr{R}_{n j}^{n^{\prime} j^{\prime}}=\frac{1}{2} \int_{0}^{\infty} d r r^{3}\left\{\left[\phi_{j^{\prime}+\uparrow}^{\left(n^{\prime}\right)}(r)\right]^{*} \phi_{j+\uparrow}^{(n)}(r)+\left[\phi_{j^{\prime}-\uparrow}^{\left(n^{\prime}\right)}(r)\right]^{*} \phi_{j-\uparrow}^{(n)}(r)\right\}
$$

for the matrix element of the radial coordinate between initial and final radial spinor wave functions from Eq. (2), and the symbols

$$
\delta_{n j, \tau}^{n^{\prime} j^{\prime}, \tau^{\prime}}=\int_{0}^{\infty} d r r^{2}\left[\phi_{j^{\prime} \tau^{\prime} \uparrow}^{\left(n^{\prime}\right)}(r)\right]^{*} \phi_{j \tau \uparrow}^{(n)}(r)
$$

are overlap integrals of radial-wave-function components.

The dependence of matrix elements given in Eqs. (6) on the angular-momentum quantum numbers of the initial and final states embodies selection rules for optical transitions between energy levels in TI nanoparticles. For states with same-type parity $\left(\kappa^{\prime}=\kappa\right)$, the rules $j^{\prime}-j= \pm 1$ and $m^{\prime}-m=0, \pm 1$ are identical to those governing atomic transitions [51]. However, there is also a previously overlooked [36] possibility for transitions between states that are of opposite type with respect to parity (i.e., $\kappa^{\prime}=-\kappa$ ), and these follow the unconventional rule $j^{\prime}-j=0$ while still adhering to $m^{\prime}-m=0, \pm 1$. As it turns out, this unconventional type of transition actually determines the low-energy threshold for optical absorption and emission in undoped TI nanoparticles.

The effect of the radial confinement on dipole-allowed single-electron transitions in TI nanoparticles is contained in the matrix elements $\mathscr{R}_{n j}^{n^{\prime} j^{\prime}}$ and the overlap integrals $\delta_{n j, \tau}^{n^{\prime} j^{\prime}, \tau^{\prime}}$ defined in Eqs. (7) and (8), respec- 

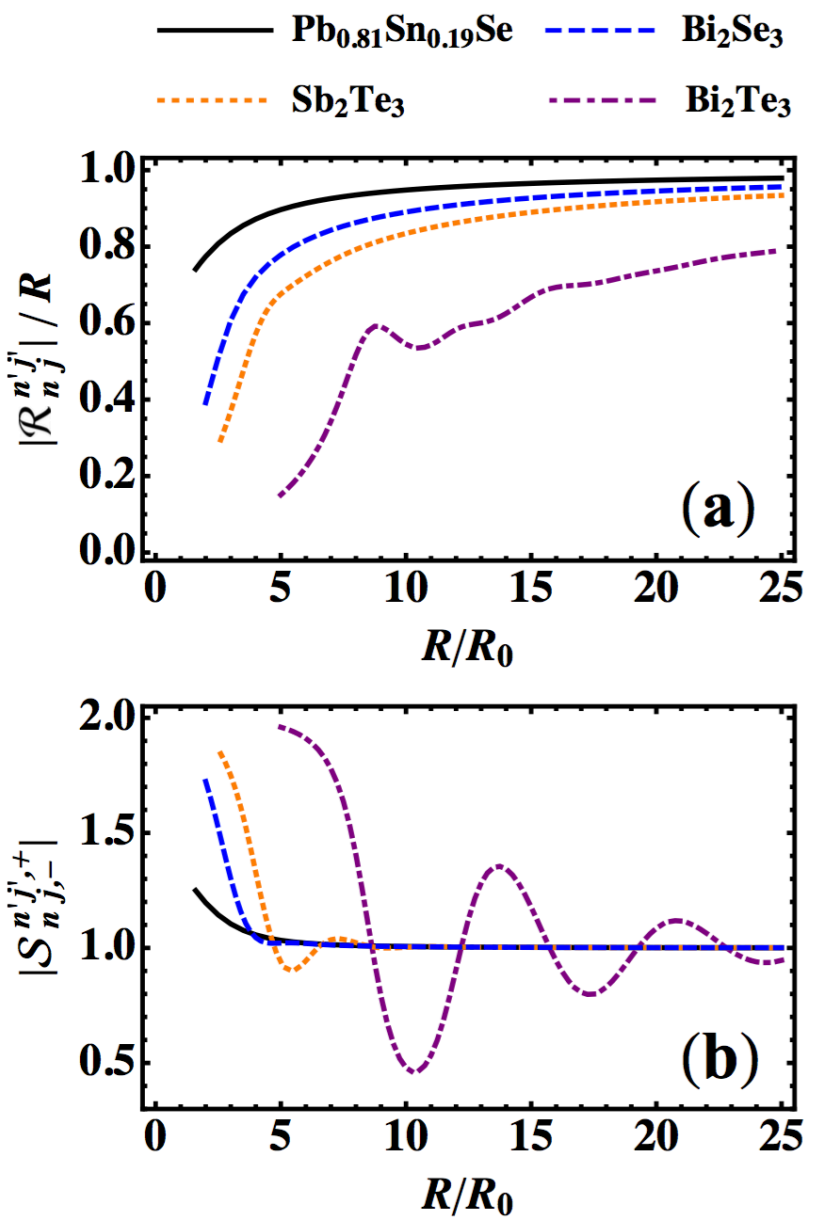

FIG. 2. Dependence of optical-transition amplitudes on TInanoparticle size. Panel (a) [(b)] shows the magnitude of the radial-coordinate matrix element $\mathscr{R}_{n j}^{n^{\prime} j^{\prime}}$ [the overlap integral $\mathcal{S}_{n j,-}^{n^{\prime} j^{\prime},+}$ for $j=j^{\prime}=1 / 2$ and $n=-1, n^{\prime}=1$, which typically corresponds to the transition across the intrinsic nanoparticle band gap, as a function of the nanoparticle radius $R$ over the range where the lowest confined electron level is still below the bulk-band-gap edge. Here $R_{0}=2 \gamma /\left|\Delta_{0}\right|$ is the bulk Compton length, and the black solid (blue dashed, orange dotted, purple dot-dashed) curve corresponds to $\Delta_{0} /\left(\gamma k_{\Delta}\right)=-0.039$ $(-1.2,-2.3,-9.5$,$) , which is the value representative of$ $\mathrm{Pb}_{0.81} \mathrm{Sn}_{0.19} \mathrm{Se}\left(\mathrm{Bi}_{2} \mathrm{Se}_{3}, \mathrm{Sb}_{2} \mathrm{Te}_{3}, \mathrm{Bi}_{2} \mathrm{Te}_{3}\right)$. From results presented in panel (b), $\left|S_{n j,+}^{n^{\prime} j^{\prime},-}\right| \equiv 2-\left|S_{n j,-}^{n^{\prime} j^{\prime},+}\right|$ can be inferred.

tively. In the large-size limit $R \gg R_{0}$, we find $\left|\mathscr{R}_{n j}^{n^{\prime} j^{\prime}}\right| \sim$ $R$ and $\left|\mathcal{S}_{n j, \mp}^{n^{\prime} j^{\prime}, \pm}\right| \sim 1$, reflecting the surface-localized massless-Dirac-fermion character of low-energy states in this regime. However, as illustrated in Figs. 2(a) and 2(b), significant deviations from this expected limiting behavior occur in the range of small-to-medium nanoparticle sizes $R \gtrsim R_{0}$. These deviations become more sizable, and also occur over a greater size range, for larger values of the TI-material parameter $-\Delta_{0} /\left(\gamma k_{\Delta}\right)$.

The remainder of this Article is structured as follows. In Section II, the continuum-model description of 3D topological insulators based on Hamiltonian (1) is used to obtain the spectrum of quantized energies and associated quantum states for a spherical hard-wall massconfinement potential. Here we derive the general form of the confined-electron eigenspinors given in Eq. (2) and also find the radial wave functions $\phi_{j \tau \sigma}^{(n)}(r)$. The formalism for describing optical properties of TI nanoparticles is developed in Section III. Transitions can be mediated by both the envelope-function part of electron wave functions and also by the Bloch-function basis states. We treat these contributions on the same footing and find a compact expression for a total optical-dipole operator that subsumes both of them [Eq. (4)]. Applying the result from Secs. II and III, we obtain the optical-transition matrix elements given in Eqs. (6). These form the basis for discussing quantum-size effects in TI nanoparticles, focusing especially on their ramifications for optical spectroscopy. Our conclusions are presented in Section IV.

\section{ELECTRON STATES IN SPHERICALLY SYMMETRIC TI NANOSTRUCTURES}

Our starting point is the effective continuum-model Hamiltonian (1), which is associated with the set of basis states given in Eq. (3). Throughout this work, we label basis states $|\tau \sigma\rangle$ in terms of pseudo-spin $\tau \in\{+,-\}$ and real spin $\sigma \in\{\uparrow, \downarrow\}$ [52]. The parameter $\gamma$ characterizes the inter-band coupling, and $\Delta_{0}$ is the energy gap of the bulk-TI material. The term involving $k_{\Delta}$ quantifies remote-band contributions that also provide a natural regularization of the leading Dirac-fermion-like dispersion that is essential for keeping the theory welldefined in the ultra-relativistic limit $\Delta_{0} \rightarrow 0$. To facilitate an instructive analytical treatment, the often quite sizeable deviations from spherical symmetry and particlehole symmetry in the TI-material bulk dispersions are omitted from the Hamiltonian (1), and materials parameters given in Table I correspond to spatial averages.

We first consider the general spherical-confinement problem in Subsection II A. Using a wave-function Ansatz involving the known eigenstates of a massless Dirac fermion moving on the surface of the unit sphere [32], we obtain a one-dimensional radial Schrödinger equation. The latter's solution for a hard-wall mass-confinement potential is presented in Subsection II B.

\section{A. Separation Ansatz and radial Schrödinger equation}

We envision the TI nanoparticle to be defined by a spherically symmetric mass-confinement potential

$$
H_{V}=V(r) \tau_{z} \otimes \sigma_{0}
$$

Then the electronic-bound-state Schrödinger equation $\left[H+H_{V}\right] \Psi=E \Psi$ is most conveniently solved by decoupling angular and radial motions. Expressing $H$ in 
terms of the usual spherical coordinates $\mathbf{r} \equiv(r, \theta, \varphi)$ and using the transformation [25]

$$
\mathcal{U}(\theta, \varphi)=\tau_{0} \otimes \exp \left(-i \frac{\varphi}{2} \sigma_{z}\right) \exp \left(-i \frac{\theta}{2} \sigma_{y}\right)
$$

it is straightforward to obtain

$$
H=\mathcal{U}(\theta, \varphi)\left(\mathscr{H}^{\perp}+\mathscr{H}^{\|}\right) \mathcal{U}^{\dagger}(\theta, \varphi)
$$

with Hamiltonians associated with radial and angular motion, respectively, given by

$$
\begin{aligned}
& \mathscr{H}^{\perp}=\left[\frac{\Delta_{0}}{2}-\frac{\gamma}{k_{\Delta}} \frac{1}{r^{2}} \partial_{r}\left(r^{2} \partial_{r}\right)\right] \tau_{z} \otimes \sigma_{0}-i \gamma\left(\partial_{r}+\frac{1}{r}\right) \tau_{x} \otimes \sigma_{z} \\
& \mathscr{H}^{\|}=\frac{\gamma}{r}\left[D \tau_{x} \otimes \sigma_{+}+D^{\dagger} \tau_{x} \otimes \sigma_{-}+\frac{1}{k_{\Delta} r}\left(D D^{\dagger} \tau_{z} \otimes \pi_{+}+D^{\dagger} D \tau_{z} \otimes \pi_{-}+i D \tau_{z} \otimes \sigma_{+}-i D^{\dagger} \tau_{z} \otimes \sigma_{-}\right)\right] .
\end{aligned}
$$

Here $\pi_{ \pm}=\left(\sigma_{0} \pm i \sigma_{z}\right) / 2$ are projection operators on the eigenstates of $\sigma_{z}$ in real-spin space, and the operators

$$
\begin{aligned}
D & =-i\left(\partial_{\theta}+\frac{\cot \theta}{2}-\frac{i \partial_{\varphi}}{\sin \theta}\right), \\
D^{\dagger} & =-i\left(\partial_{\theta}+\frac{\cot \theta}{2}+\frac{i \partial_{\varphi}}{\sin \theta}\right)
\end{aligned}
$$

appear in the Dirac operator on the unit sphere [32].

The spherical mass-confinement potential $H_{V}$ trivially commutes with the transformation $\mathcal{U}(\theta, \varphi)$, i.e., $\mathscr{H}_{V} \equiv$ $\mathcal{U}^{\dagger}(\theta, \varphi) H_{V} \mathcal{u}(\theta, \varphi)=H_{V}$. This feature, together with the structure of the angular-motion Hamiltonian $\mathscr{H}^{\|}$, mo- tivates the separation Ansatz

$$
\Psi(r, \theta, \varphi)=\mathcal{U}(\theta, \varphi) \frac{\mathrm{e}^{i m \varphi}}{\sqrt{4 \pi}}\left(\begin{array}{c}
\alpha_{\lambda m}(\theta) \phi_{|\lambda|+\uparrow}(r) \\
\alpha_{\lambda m}(\theta) \phi_{|\lambda|-\uparrow}(r) \\
\beta_{\lambda m}(\theta) \phi_{|\lambda|+\downarrow}(r) \\
\beta_{\lambda m}(\theta) \phi_{|\lambda|-\downarrow}(r)
\end{array}\right)
$$

where the functions $\alpha_{\lambda m}(\theta)$ and $\beta_{\lambda m}(\theta)$ are the polarangle-dependent entries in eigenspinors of a massless Dirac fermion moving on a spherical shell [25, 32]. Using the fact that they satisfy the set of relations

$$
\begin{gathered}
D \beta_{\lambda m}(\theta)=\lambda \alpha_{\lambda m}(\theta), D^{\dagger} \alpha_{\lambda m}(\theta)=\lambda \beta_{\lambda m}(\theta) \\
\alpha_{\lambda m}=\left(\frac{\lambda}{|\lambda|}\right)^{\frac{1}{2}} \alpha_{|\lambda| m}, \beta_{\lambda m}=\left(\frac{\lambda}{|\lambda|}\right)^{-\frac{1}{2}} \beta_{|\lambda| m},
\end{gathered}
$$

we obtain the radial Schrödinger equation

$$
\left[\mathscr{H}_{|\lambda|}^{\perp}+\mathscr{H}_{V}\right] \Phi_{|\lambda|}(r)=E_{|\lambda|} \Phi_{|\lambda|}(r)
$$

for the spinor $\Phi_{|\lambda|}=\left(\phi_{|\lambda|+\uparrow}, \phi_{|\lambda|-\uparrow}, \phi_{|\lambda|+\downarrow}, \phi_{|\lambda|-\downarrow}\right)^{T}$, with the free radial Hamiltonian given by

$$
\mathscr{H}_{|\lambda|}^{\perp}=\left\{\frac{\Delta_{0}}{2}+\frac{\gamma}{k_{\Delta}} \frac{1}{r^{2}}\left[|\lambda|^{2}-\partial_{r}\left(r^{2} \partial_{r}\right)\right]\right\} \tau_{z} \otimes \sigma_{0}-i \gamma\left(\partial_{r}+\frac{1}{r}\right) \tau_{x} \otimes \sigma_{z}+\frac{\gamma}{r}|\lambda|\left(\tau_{x} \otimes \sigma_{x}-\frac{1}{k_{\Delta} r} \tau_{z} \otimes \sigma_{y}\right)
$$

As previously discussed, e.g., in Ref. [32], $|\lambda|$ is related to the half-integer eigenvalue $j$ of total angular momentum $\mathbf{J} \equiv\left(\mathbf{L}+\frac{\hbar}{2} \boldsymbol{\sigma}\right)$ via $|\lambda|=j+\frac{1}{2}$ and therefore must be a nonzero integer. The quantum numbers $m=-j,-j+1, \ldots j$ are the eigenvalues of the angularmomentum projection and, thus, also assume half-integer values. With this additional insight, solution of the radial Schrödinger equation (16) can proceed for any spe- cific potential $V(r)$. Because of the one-to-one correspondence between values of $|\lambda|$ and $j$, we can use both interchangeably as labels for radial spinor wave functions; $\Phi_{|\lambda|}(r) \equiv \Phi_{j}(r)$, and its components $\phi_{|\lambda| \tau \sigma}(r) \equiv \phi_{j \tau \sigma}(r)$. In the following Subsection IIB, we consider the special case of a hard-wall confinement. Using results obtained there as input, the electronic eigenstates are found to be of the form 


$$
\begin{aligned}
& \Psi_{\lambda m}^{\left(n_{\mathrm{a}}\right)}(\mathbf{r})=\mathcal{U}(\theta, \varphi) \frac{\mathrm{e}^{i m \varphi}}{\sqrt{4 \pi}}\left\{\left(\begin{array}{c}
\alpha_{\lambda m}(\theta) \\
0 \\
i \beta_{\lambda m}(\theta) \\
0
\end{array}\right) \phi_{j+\uparrow}^{\left(n_{\mathrm{a}}\right)}(r)+\left(\begin{array}{c}
0 \\
\alpha_{\lambda m}(\theta) \\
0 \\
-i \beta_{\lambda m}(\theta)
\end{array}\right) \phi_{j-\uparrow}^{\left(n_{\mathrm{a}}\right)}(r)\right\}, \\
& \Psi_{\lambda m}^{\left(n_{\mathrm{b}}\right)}(\mathbf{r})=\mathcal{U}(\theta, \varphi) \frac{\mathrm{e}^{i m \varphi}}{\sqrt{4 \pi}}\left\{\left(\begin{array}{c}
\alpha_{\lambda m}(\theta) \\
0 \\
-i \beta_{\lambda m}(\theta) \\
0
\end{array}\right) \phi_{j+\uparrow}^{\left(n_{\mathrm{b}}\right)}(r)+\left(\begin{array}{c}
0 \\
\alpha_{\lambda m}(\theta) \\
0 \\
i \beta_{\lambda m}(\theta)
\end{array}\right) \phi_{j-\uparrow}^{\left(n_{\mathrm{b}}\right)}(r)\right\} .
\end{aligned}
$$

Here $n_{\mathrm{a}}$ and $n_{\mathrm{b}}$ are the radial-quantum-number labels for two sets of energy levels arising from two distinct secular equations. Adapting the approach presented in Ref. [32], it is possible to express the wave functions (18a) and (18b) in terms of spherical harmonics. This yields the results presented in Eq. (2) above, where

$$
\begin{array}{ll}
\Psi_{\lambda m}^{(n)}(\mathbf{r}) \equiv \Psi_{j m+}^{(n)}(\mathbf{r}) & \text { for } n \in\left\{n_{\mathrm{a}}\right\} \text { and } \lambda>0 \\
& \text { or } n \in\left\{n_{\mathrm{b}}\right\} \text { and } \lambda<0 \\
\Psi_{\lambda m}^{(n)}(\mathbf{r}) \equiv \Psi_{j m-}^{(n)}(\mathbf{r}) & \text { for } n \in\left\{n_{\mathrm{a}}\right\} \text { and } \lambda<0 \\
& \text { or } n \in\left\{n_{\mathrm{b}}\right\} \text { and } \lambda>0
\end{array},
$$

and prefactors given by

$$
C_{j \kappa}^{(n)}=\left\{\begin{array}{cl}
i^{j} & n \in\left\{n_{\mathrm{a}}\right\}, \kappa=+ \text { or } n \in\left\{n_{\mathrm{b}}\right\}, \kappa=- \\
-i^{j+1} & n \in\left\{n_{\mathrm{a}}\right\}, \kappa=- \text { or } n \in\left\{n_{\mathrm{b}}\right\}, \kappa=+
\end{array} .\right.
$$

Our route to obtain (2) via the Ansatz (14) is inspired by a particular way of solving the Dirac equation on a spherical shell $[25,30,32]$, but this method is far from unique. An equivalent radial Schrödinger equation and eigenstates having the same angular-coordinate dependences as those in (2) should transpire, e.g., from writing Ansätze for eigenstates of the original Hamiltonian (1) in terms of spinor spherical harmonics [53] and applying the mathematical identities satisfied by them [54].

\section{B. Solution of the radial Schrödinger equation for a hard-wall mass confinenment}

Solution of the radial Schrödinger equation (16) for a hard-wall mass confinement defining a TI nanoparticle with radius $R$ requires obtaining eigenstates of $\mathscr{H}_{j+\frac{1}{2}}^{\perp}$ from Eq. (17) and forming linear combinations of these for fixed energy $E$ that satisfy the hard-wall boundary condition at $r=R$. The secular equation associated with the linear system of equations for the superposition coefficients yields the bound-state energies.

There is a set of propagating-wave-like eigenstates for $\mathscr{H}_{j+\frac{1}{2}}^{\perp}$ that have the general form

$$
\Phi_{j, \mathrm{a}}^{(\mathrm{D})}(r)=\left(\begin{array}{c}
j_{j-\frac{1}{2}}(k r) \\
i \gamma_{k} j_{j+\frac{1}{2}}(k r) \\
i j_{j-\frac{1}{2}}(k r) \\
\gamma_{k} j_{j+\frac{1}{2}}(k r)
\end{array}\right)
$$

$$
\Phi_{j, \mathrm{~b}}^{(\mathrm{D})}(r)=\left(\begin{array}{c}
j_{j+\frac{1}{2}}(k r) \\
-i \gamma_{k} j_{j-\frac{1}{2}}(k r) \\
-i j_{j+\frac{1}{2}}(k r) \\
\gamma_{k} j_{j-\frac{1}{2}}(k r)
\end{array}\right),
$$

where $j_{\nu}(\cdot)$ are spherical Bessel functions of the first kind [55] (not to be confused with the quantum number $j$ of total angular momentum), $k>0$ is a real number, and normalization factors have been omitted because they get absorbed into the coefficients in the bound-state linear combination. The energy eigenvalues are given by

$$
\tilde{E}_{ \pm}^{(\mathrm{D})}(k)= \pm \sqrt{\tilde{k}^{4}+\left(1+\tilde{\Delta}_{0}\right) \tilde{k}^{2}+\frac{\tilde{\Delta}_{0}^{2}}{4}}
$$

and the parameter entering corresponding eigenspinors can be found from the general expression

$$
\gamma_{k}=\operatorname{sgn}(E) \sqrt{\frac{2 \tilde{E}-\tilde{\Delta}_{0}-2 \tilde{k}^{2}}{2 \tilde{E}+\tilde{\Delta}_{0}+2 \tilde{k}^{2}}} .
$$

Here we started using natural units for energies and wave numbers via the definitions $\tilde{k}=k / k_{\Delta}$ and $\tilde{E}=$ $E /\left(\gamma k_{\Delta}\right)$. The particular form of the eigenstates given in Eqs. (21) is reminiscent of confined-Dirac-particle states $[23,35,56]$. The absence of spherical Bessel functions of the second kind from the wave-function Ansatz (21) is dictated by the necessity of wave functions to be well-behaved at $r=0$.

There exists another set of eigenstates for $\mathscr{H}_{j+\frac{1}{2}}^{\perp}$ that are evanescent in character;

$$
\begin{gathered}
\Phi_{j, \mathrm{a}}^{(\mathrm{B})}(r)=\frac{1}{\sqrt{q r}}\left(\begin{array}{c}
\bar{\gamma}_{q} I_{j}(q r) \\
i I_{j+1}(q r) \\
i \bar{\gamma}_{q} I_{j}(q r) \\
I_{j+1}(q r)
\end{array}\right), \\
\Phi_{j, \mathrm{~b}}^{(\mathrm{B})}(r)=\frac{1}{\sqrt{q r}}\left(\begin{array}{c}
-\bar{\gamma}_{q} I_{j+1}(q r) \\
-i I_{j}(q r) \\
i \bar{\gamma}_{q} I_{j+1}(q r) \\
I_{j}(q r)
\end{array}\right),
\end{gathered}
$$

where the spinor entries are modified spherical Bessel functions of the first kind [55], and $q>0$ is real. The associated energy eigenvalues are

$$
\tilde{E}_{ \pm}^{(\mathrm{B})}(q)= \pm \sqrt{\tilde{q}^{4}-\left(1+\tilde{\Delta}_{0}\right) \tilde{q}^{2}+\frac{\tilde{\Delta}_{0}^{2}}{4}}
$$


and

$$
\bar{\gamma}_{q}=\sqrt{\frac{2 \tilde{q}^{2}-\tilde{\Delta}_{0}-2 \tilde{E}}{2 \tilde{q}^{2}-\tilde{\Delta}_{0}+2 \tilde{E}}} .
$$

The consideration of such evanescent free-particle eigenstates in the context of BHZ-type model Hamiltonians $[18,57]$ is required for mathematical consistency, but their physical significance is limited [58-60].

Having determined the eigenstates of $\mathscr{H}_{j+\frac{1}{2}}^{\perp}$, we can make an Ansatz for the wave function of hard-wallconfined electrons in the TI nanoparticle in the form of superpositions

$$
\begin{aligned}
\Phi_{j}^{(n)}(r)=c_{n, j, \mathrm{a}}^{(\mathrm{D})} \Phi_{j, \mathrm{a}}^{(\mathrm{D})}(r)+c_{n, j, \mathrm{a}}^{(\mathrm{B})} \Phi_{j, \mathrm{a}}^{(\mathrm{B})}(r) & \\
& +c_{n, j, \mathrm{~b}}^{(\mathrm{D})} \Phi_{j, \mathrm{~b}}^{(\mathrm{D})}(r)+c_{n, j, \mathrm{~b}}^{(\mathrm{B})} \Phi_{j, \mathrm{~b}}^{(\mathrm{B})}(r)
\end{aligned}
$$

of such eigenstates for fixed energy $E$ and require that the boundary condition $\Phi_{j}^{(n)}(R)=0$ is satisfied. The associated system of linear equations for the coefficients $c_{n, j, \mathrm{a} / \mathrm{b}}^{(\mathrm{D}, \mathrm{B})}$ has nontrivial solutions only if one of the secular equations

$$
\gamma_{k} \bar{\gamma}_{q}=\left\{\begin{aligned}
\frac{I_{j+1}(q R)}{I_{j}(q R)} & \frac{j_{j-\frac{1}{2}}(k R)}{j_{j+\frac{1}{2}}(k R)} \\
-\frac{I_{j}(q R)}{I_{j+1}(q R)} & \text { case a } \\
j_{j-\frac{1}{2}}(k R) & \text { case b },
\end{aligned}\right.
$$

is fulfilled. In Eq. (28), the quantities $k, q, \gamma_{k}$ and $\bar{\gamma}_{q}$ are functions of $E$, given explicitly by [61]

$$
\begin{aligned}
\tilde{k} & =\left[\frac{1}{2}\left(\sqrt{\left(1+\tilde{\Delta}_{0}\right)^{2}+4 \tilde{E}^{2}-\tilde{\Delta}_{0}^{2}}-1-\tilde{\Delta}_{0}\right)\right]^{\frac{1}{2}}, \\
\tilde{q} & =\left[\frac{1}{2}\left(\sqrt{\left(1+\tilde{\Delta}_{0}\right)^{2}+4 \tilde{E}^{2}-\tilde{\Delta}_{0}^{2}}+1+\tilde{\Delta}_{0}\right)\right]^{\frac{1}{2}},(29 \\
\gamma_{k} & =\operatorname{sgn}(\tilde{E})\left[\frac{2 \tilde{E}-\sqrt{\left(1+\tilde{\Delta}_{0}\right)^{2}+4 \tilde{E}^{2}-\tilde{\Delta}_{0}^{2}}+1}{2 \tilde{E}+\sqrt{\left(1+\tilde{\Delta}_{0}\right)^{2}+4 \tilde{E}^{2}-\tilde{\Delta}_{0}^{2}}-1}\right]^{\frac{1}{2}}, \\
\bar{\gamma}_{q} & =\left[\frac{\sqrt{\left(1+\tilde{\Delta}_{0}\right)^{2}+4 \tilde{E}^{2}-\tilde{\Delta}_{0}^{2}}+1-2 \tilde{E}}{\sqrt{\left(1+\tilde{\Delta}_{0}\right)^{2}+4 \tilde{E}^{2}-\tilde{\Delta}_{0}^{2}}+1+2 \tilde{E}}\right]^{\frac{1}{2}} .
\end{aligned}
$$

Solution of Eq. (28) for cases a and b yields associated series of quantized energy values $E_{j}^{\left(n_{\mathrm{a}}\right)}$ and $E_{j}^{\left(n_{\mathrm{b}}\right)}$, respectively. By construction, these satisfy $\left|E_{j}^{\left(n_{\mathrm{a} / \mathrm{b}}\right)}\right| \geq\left|\Delta_{0}\right| / 2$. The coefficients in the superposition (27) of corresponding bound states are straightforwardly found to be

$$
\begin{aligned}
c_{n_{\mathrm{a}}, j, \mathrm{a}}^{(\mathrm{D})}=N_{n_{\mathrm{a}}, j}, c_{n_{\mathrm{a}}, j, \mathrm{a}}^{(\mathrm{B})}= & N_{n_{\mathrm{a}}, j} \xi_{n_{\mathrm{a}}, j, \mathrm{a}}, \\
& c_{n_{\mathrm{a}}, j, \mathrm{~b}}^{(\mathrm{D})}=c_{n_{\mathrm{a}}, j, \mathrm{~b}}^{(\mathrm{B})}=0, \\
c_{n_{\mathrm{b}}, j, \mathrm{~b}}^{(\mathrm{D})}=N_{n_{\mathrm{b}}, j}, c_{n_{\mathrm{b}}, j, \mathrm{~b}}^{(\mathrm{B})}= & N_{n_{\mathrm{b}}, j} \xi_{n_{\mathrm{b}}, j, \mathrm{~b}},
\end{aligned}
$$

$$
c_{n_{\mathrm{b}}, j, \mathrm{a}}^{(\mathrm{D})}=c_{n_{\mathrm{b}}, j, \mathrm{a}}^{(\mathrm{B})}=0,
$$

with normalisation factors $N_{n_{\mathrm{a} / \mathrm{b}}, j}$ and the parameters

$$
\begin{aligned}
& \xi_{n_{\mathrm{a}}, j, \mathrm{a}}=-\left.\frac{\gamma_{k} \sqrt{q R} j_{j+\frac{1}{2}}(k R)}{I_{j+1}(q R)}\right|_{E=E_{j}^{\left(n_{\mathrm{a}}\right)}}, \\
& \xi_{n_{\mathrm{b}}, j, \mathrm{~b}}=-\left.\frac{\gamma_{k} \sqrt{q R} j_{j-\frac{1}{2}}(k R)}{I_{j}(q R)}\right|_{E=E_{j}^{\left(n_{\mathrm{b}}\right)}} .
\end{aligned}
$$

Motivated by the well-known $[18,23,57,62]$ existence of evanescent states having $|E|<\left|\Delta_{0}\right| / 2$ in the topological regime [63] where $\Delta_{0}<0$, we consider also the modified Ansatz

$$
\begin{aligned}
\Phi_{j}^{(n)}(r)=c_{n, j, \mathrm{a}}^{(\mathrm{P})} & \Phi_{j, \mathrm{a}}^{(\mathrm{P})}(r)+c_{n, j, \mathrm{a}}^{(\mathrm{B})} \Phi_{j, \mathrm{a}}^{(\mathrm{B})}(r) \\
& +c_{n, j, \mathrm{~b}}^{(\mathrm{P})} \Phi_{j, \mathrm{~b}}^{(\mathrm{P})}(r)+c_{n, j, \mathrm{~b}}^{(\mathrm{B})} \Phi_{j, \mathrm{~b}}^{(\mathrm{B})}(r),
\end{aligned}
$$

which is obtained by replacing the propagating-wave-like part $c_{n, j, \mathrm{a} / \mathrm{b}}^{(\mathrm{D})} \Phi_{j, \mathrm{a} / \mathrm{b}}^{(\mathrm{D})}(r)$ in Eq. $(27)$ by $c_{n, j, \mathrm{a} / \mathrm{b}}^{(\mathrm{P})} \Phi_{j, \mathrm{a} / \mathrm{b}}^{(\mathrm{P})}(r)$ with the evanescent-wave spinors [64]

$$
\begin{gathered}
\Phi_{j, \mathrm{a}}^{(\mathrm{P})}(r)=\frac{1}{\sqrt{\bar{k} r}}\left(\begin{array}{c}
I_{j}(\bar{k} r) \\
-i \bar{\gamma}_{\bar{k}} I_{j+1}(\bar{k} r) \\
i I_{j}(\bar{k} r) \\
-\bar{\gamma}_{\bar{k}} I_{j+1}(\bar{k} r)
\end{array}\right), \\
\Phi_{j, \mathrm{~b}}^{(\mathrm{P})}(r)=\frac{1}{\sqrt{\bar{k} r}}\left(\begin{array}{c}
i I_{j+1}(\bar{k} r) \\
\bar{\gamma}_{\bar{k}} I_{j}(\bar{k} r) \\
I_{j+1}(\bar{k} r) \\
i \bar{\gamma}_{\bar{k}} I_{j}(\bar{k} r)
\end{array}\right) .
\end{gathered}
$$

The energy-dependent parameters entering Eqs. (32) are

$$
\begin{gathered}
\tilde{\bar{k}}=\left[\frac{1}{2}\left(1+\tilde{\Delta}_{0}-\sqrt{\left(1+\tilde{\Delta}_{0}\right)^{2}+4 \tilde{E}^{2}-\tilde{\Delta}_{0}^{2}}\right)\right]^{\frac{1}{2}},(33 \mathrm{a}) \\
\bar{\gamma}_{\bar{k}}=\operatorname{sgn}\left(\Delta_{0}\right)\left[\frac{\sqrt{\left(1+\tilde{\Delta}_{0}\right)^{2}+4 \tilde{E}^{2}-\tilde{\Delta}_{0}^{2}}-1-2 \tilde{E}}{\sqrt{\left(1+\tilde{\Delta}_{0}\right)^{2}+4 \tilde{E}^{2}-\tilde{\Delta}_{0}^{2}}-1+2 \tilde{E}}\right]^{\frac{1}{2}} .
\end{gathered}
$$

Imposing the hard-wall boundary condition at $r=R$ yields the secular equations

$$
-\bar{\gamma}_{\bar{k}} \bar{\gamma}_{q}= \begin{cases}\frac{I_{j+1}(q R)}{I_{j}(q R)} \frac{I_{j}(\bar{k} R)}{I_{j+1}(\bar{k} R)} & \text { case a } \\ \frac{I_{j}(q R)}{I_{j+1}(q R)} \frac{I_{j+1}(\bar{k} R)}{I_{j}(k R)} & \text { case b. }\end{cases}
$$

The coefficients in the Ansatz (31) involving only evanescent eigenstates of $\mathscr{H}_{j+\frac{1}{2}}^{\perp}$ are

$$
\begin{aligned}
c_{n_{\mathrm{a}}, j, \mathrm{a}}^{(\mathrm{P})}=\bar{N}_{n_{\mathrm{a}}, j}, c_{n_{\mathrm{a}}, j, \mathrm{a}}^{(\mathrm{B})}= & \bar{N}_{n_{\mathrm{a}}, j} \bar{\xi}_{n_{\mathrm{a}}, j, \mathrm{a}}, \\
& c_{n_{\mathrm{a}}, j, \mathrm{~b}}^{(\mathrm{P})}=c_{n_{\mathrm{a}}, j, \mathrm{~b}}^{(\mathrm{B})}=0,
\end{aligned}
$$




$$
\begin{aligned}
c_{n_{\mathrm{b}}, j, \mathrm{~b}}^{(\mathrm{P})}=\bar{N}_{n_{\mathrm{b}}, j}, c_{n_{\mathrm{b}}, j, \mathrm{~b}}^{(\mathrm{B})}= & \bar{N}_{n_{\mathrm{b}}, j} \bar{\xi}_{n_{\mathrm{b}}, j, \mathrm{~b}}, \\
& c_{n_{\mathrm{b}}, j, \mathrm{a}}^{(\mathrm{P})}=c_{n_{\mathrm{b}}, j, \mathrm{a}}^{(\mathrm{B})}=0,
\end{aligned}
$$

with the parameters

$$
\begin{aligned}
& \bar{\xi}_{n_{\mathrm{a}}, j, \mathrm{a}}=\left.\bar{\gamma}_{\bar{k}} \sqrt{\frac{q}{\bar{k}}} \frac{I_{j+1}(\bar{k} R)}{I_{j+1}(q R)}\right|_{E=E_{j}^{\left(n_{\mathrm{a}}\right)}}, \\
& \bar{\xi}_{n_{\mathrm{b}}, j, \mathrm{~b}}=-\left.i \overline{\gamma_{\bar{k}}} \sqrt{\frac{q}{\bar{k}}} \frac{I_{j}(\bar{k} R)}{I_{j}(q R)}\right|_{E=E_{j}^{\left(n_{\mathrm{b}}\right)}} .
\end{aligned}
$$

The general form (14) of TI-nanoparticle bound-state wave functions, together with the Ansätze (27) and (31) [using also (21), (24), and (32)] implies that the electron eigenstates in TI nanoparticles can be written as given in Eqs. (18a) and (18b). These expressions form the basis for deriving Eq. (2), which is one of the main results of this work. We use the general convention where energy levels for fixed $j$ have labels $n$ such that $E_{j}^{\left(n^{\prime}\right)}>E_{j}^{(n)}$ for $n^{\prime}>n$ and $n>0(n<0)$ if $E_{j}^{(n)}>0\left(E_{j}^{(n)}<0\right)$. In addition to the quantized energies among any fixed- $j$ series that arise as solutions of the secular equations (28), (34) yields two more bound-state levels with energies within the bulk-material band gap when $\Delta_{0}$ is negative and $j$ below an $R$-dependent critical value $j_{\mathrm{c}}$. For large $R, j_{\mathrm{c}}$ is also large, and the many solutions of (34) correspond to the topologically protected surface states of a bulkTI material. Reducing $R$ successively eliminates such solutions for higher $j$. Eventually, at $R=R_{\mathrm{c}}$, the energies of the $j=1 / 2$ sub-gap bound states are pushed beyond $\pm\left|\Delta_{0}\right| / 2$ and the TI-nanoparticle spectrum becomes non-inverted. Figure 1 illustrates this general behavior. Assuming $q R \gg 1$ (which is typically satisfied) and $\Delta_{0} /\left(\gamma k_{\Delta}\right)>-1 / 2$ (which can be violated in existing materials), we find the analytical expressions

$$
\begin{aligned}
j_{\mathrm{c}} & =\left\lfloor\sqrt{1+\frac{\Delta_{0}}{\gamma k_{\Delta}}} \frac{R}{R_{0}}-1\right\rfloor, \\
R_{\mathrm{c}} & =\frac{3}{2} \frac{R_{0}}{\sqrt{1+\Delta_{0} /\left(\gamma k_{\Delta}\right)}} .
\end{aligned}
$$

\section{OPTICAL TRANSITIONS BETWEEN BOUND STATES IN TI NANOPARTICLES}

Optical spectroscopy provides a useful tool to study the electronic structure of semiconductor nanomaterials [65, 66], including single nanoparticles [67] and their ensembles [68]. In the following, we explore how the special properties of TI nanoparticles are manifested in their optical spectra. We start by discussing the general theoretical approach for treating confined multi-band systems and then focus on our particular system of interest.

Electron states in the TI nanoparticle have the form

$$
\left|\Psi_{j m \kappa}^{(n)}(\mathbf{r})\right\rangle=\sum_{\tau \sigma}\left[\Psi_{j m \kappa}^{(n)}(\mathbf{r})\right]_{\tau \sigma}|\tau \sigma\rangle
$$

where the $|\tau \sigma\rangle$ are the band-edge basis functions [37-39] listed in Eq. (3), and $\left[\Psi_{j m \kappa}^{(n)}(\mathbf{r})\right]_{\tau \sigma}$ denote entries in the envelope-function spinor $\Psi_{j m \kappa}^{(n)}(\mathbf{r})$ given in Eq. (2). Optical transitions of electrons in this system are mediated by the dipole matrix element, which is most generally given as the sum of two contributions [69];

$$
\mathbf{d}_{n j m \kappa}^{n^{\prime} j^{\prime} m^{\prime} \kappa^{\prime}}=\mathscr{D}_{n j m \kappa}^{n^{\prime} j^{\prime} m^{\prime} \kappa^{\prime}}+\mathscr{B}_{n j m \kappa}^{n^{\prime} j^{\prime} m^{\prime} \kappa^{\prime}}
$$

where

$$
\mathscr{D}_{n j m \kappa}^{n^{\prime} j^{\prime} m^{\prime} \kappa^{\prime}}=\sum_{\tau \sigma} \int d^{3} r\left[\Psi_{j^{\prime} m^{\prime} \kappa^{\prime}}^{\left(n^{\prime}\right)}(\mathbf{r})\right]_{\tau \sigma}^{*} e \mathbf{r}\left[\Psi_{j m \kappa}^{(n)}(\mathbf{r})\right]_{\tau \sigma}
$$

is the dipole matrix element for the envelope part of the electronic wave functions ( $e$ is the electron charge), and

$$
\mathscr{B}_{n j m \kappa}^{n^{\prime} j^{\prime} m^{\prime} \kappa^{\prime}}=\sum_{\substack{\tau \tau \prime \\ \sigma \sigma^{\prime}}} \mathscr{F}_{n j m \kappa \tau \sigma}^{n^{\prime} j^{\prime} m^{\prime} \kappa^{\prime} \tau^{\prime} \sigma^{\prime}} \mathbf{d}_{\tau \sigma}^{\tau^{\prime} \sigma^{\prime}}
$$

contains the contributions of dipole matrix elements $\mathbf{d}_{\tau \sigma}^{\tau^{\prime} \sigma^{\prime}} \equiv\left\langle\tau^{\prime} \sigma^{\prime}|e \mathbf{r}| \tau \sigma\right\rangle$ between band-edge basis states, which are renormalized by the form factors

$$
\mathscr{F}_{n j m \kappa \tau \sigma}^{n^{\prime} j^{\prime} m^{\prime} \kappa^{\prime} \tau^{\prime} \sigma^{\prime}}=\int d^{3} r\left[\Psi_{j^{\prime} m^{\prime} \kappa^{\prime}}^{\left(n^{\prime}\right)}(\mathbf{r})\right]_{\tau^{\prime} \sigma^{\prime}}^{*}\left[\Psi_{j m \kappa}^{(n)}(\mathbf{r})\right]_{\tau \sigma} .
$$

When optical properties of wide-band-gap semiconductor materials are discussed, the basis-function-related contributions $\mathscr{B}_{n j m \kappa}^{n^{\prime} j^{\prime} m^{\prime} \kappa^{\prime}}$ are understood to represent 'interband' transitions [65] occurring between states from the valence and conduction bands. In contrast, the envelope-function contributions $\mathscr{D}_{n j m \kappa}^{n^{\prime} j^{\prime} m^{\prime} \kappa^{\prime}}$ in such systems are referred to as 'intraband' or 'intersubband' because they involve initial and final states that are sizequantized levels deriving from the same (either conduction or valence) band. In our present case of interest, these two types of contributions to the optical matrix element cannot be neatly classified as either 'interband' or 'intraband' anymore, as the amplitude of every possible transition will be influenced significantly by both [36].

The fundamental relationship [65] between optical matrix elements for band-edge basis functions and linear-in$\mathbf{k}$ terms in the $\mathbf{k} \cdot \mathbf{p}$ Hamiltonian Eq. (1) implies

$$
\mathbf{d}_{\tau \sigma}^{\tau^{\prime} \sigma^{\prime}}=\tau \frac{i e}{\Delta_{0}}\left(\left.\nabla_{\mathbf{k}} H\right|_{\mathbf{k}=\mathbf{0}}\right)_{\tau \sigma}^{\tau^{\prime} \sigma^{\prime}}
$$

where we use the notation $(O)_{\tau \sigma}^{\tau^{\prime} \sigma^{\prime}}$ to indicate matrix elements of an operator $O$ acting in $\mathbf{k} \cdot \mathbf{p}$ space. Using (42), we can conveniently rewrite the basis-function contribution to the optical dipole matrix element as

$$
\mathscr{B}_{n j m \kappa}^{n^{\prime} j^{\prime} m^{\prime} \kappa^{\prime}} \equiv \frac{e R_{0}}{2} \int d^{3} r\left[\Psi_{j^{\prime} m^{\prime} \kappa^{\prime}}^{\left(n^{\prime}\right)}(\mathbf{r})\right]^{\dagger} \tau_{y} \otimes \boldsymbol{\sigma} \Psi_{j m \kappa}^{(n)}(\mathbf{r}) .
$$

Together with (39), we can thus express the total dipole matrix element for optical transitions entirely within 


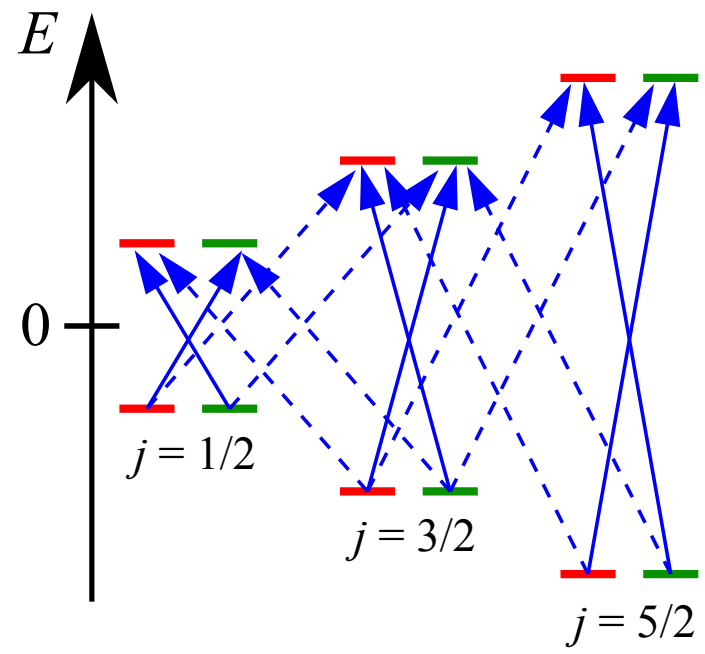

FIG. 3. Optical transitions in undoped TI nanoparticles between levels derived from topologically protected surface states. Red (green) color indicates states with parity-related quantum number $\kappa=+(-)$. Transitions conserving $\kappa$ (illustrated by dashed-line arrows) require a change in the total-angular-momentum quantum number $j$ by \pm 1 . Transitions conserving $j$ (indicated by solid-line arrows) are possible between states having opposite $\kappa$. The selection rule $\Delta m=0, \pm 1$ applies to all of these transitions.

envelope-function space as shown in Eq. (5), with the operator d given in Eq. (4).

In the calculation of the envelope-function part (39) of the optical-dipole matrix element (38), we employ the standard [51] representation of real-space coordinates in terms of spherical harmonics,

$$
\begin{aligned}
x \pm i y & =\mp \sqrt{\frac{8 \pi}{3}} r Y_{1}^{ \pm 1}(\theta, \varphi), \\
z & =\sqrt{\frac{4 \pi}{3}} r Y_{1}^{0}(\theta, \varphi) .
\end{aligned}
$$

We also apply a well-known integral identity for spherical harmonics [see, e.g., Eq. (3.8.73) from Ref. [46]) to obtain

$$
\begin{aligned}
& \int_{0}^{\pi} d \theta \sin \theta \int_{0}^{2 \pi} d \varphi\left[Y_{l^{\prime}}^{m^{\prime}}(\theta, \varphi)\right]^{*} Y_{1}^{m_{1}}(\theta, \varphi) Y_{l}^{m}(\theta, \varphi) \\
& =\sqrt{\frac{3}{4 \pi}} \sqrt{\frac{2 l+1}{2 l^{\prime}+1}}\left\langle l 0 ; 10 \mid l^{\prime} 0\right\rangle\left\langle l m ; 1 m_{1} \mid l^{\prime} m^{\prime}\right\rangle,
\end{aligned}
$$

where the symbol $\left\langle l_{1} m_{1} ; l_{2} m_{2} \mid l m\right\rangle$ is used to denote Clebsch-Gordon coefficients [46]. Algebraic simplification then yields the terms in Eqs. (6) that are proportional to $\mathscr{R}_{n j}^{n^{\prime} j^{\prime}}$. Similarly, the terms in (6) that are proportional to the overlap integrals $\delta_{n j, \tau}^{n^{\prime} j^{\prime}, \tau^{\prime}}$ are obtained from calculating the basis-function contribution (43) to the optical-dipole matrix element (38) using the orthogonality relation for spherical harmonics.

Results from Sec. II imply that in-gap bound states from the case-a series have eigenenergies with equal magnitude but opposite sign as those of the case-b series. Each of these levels is degenerate in the parity-related quantum number $\kappa$. Hence, in an undoped TI nanoparticle, optical transitions can occur between the oppositeenergy eigenstates, and the energy cutoff for photon absorption and emission via single-electron transitions is given by the TI-nanoparticle band gap $2 \cdot \min \left\{E_{j}^{(1)}\right\}$. We illustrate the allowed optical transitions between topological (i.e., in-gap) nanoparticle bound states in Fig. 3. Such single-particle transitions will dominantly determine the optical properties of nanoparticles with sizes smaller than the charge-carriers' Bohr radius [70]. The total magnitude of optical transition matrix elements associated with such transitions is given by

$$
\begin{aligned}
& \left(T_{ \pm}\right)_{n j \kappa}^{n^{\prime} j^{\prime} \kappa^{\prime}}=\sum_{m=-j}^{j}\left|\left(d_{x} \pm i d_{y}\right)_{n j m \kappa}^{n^{\prime} j^{\prime} m^{\prime} \kappa^{\prime}}\right|^{2} \equiv \frac{2 e^{2} R^{2}}{3} \mathscr{T}_{n j \kappa}^{n^{\prime} j^{\prime} \kappa^{\prime}}, \\
& \left(T_{z}\right)_{n j \kappa}^{n^{\prime} j^{\prime} \kappa^{\prime}}=\sum_{m=-j}^{j}\left|\left(d_{z}\right)_{n j m \kappa}^{n^{\prime} j^{\prime} m^{\prime} \kappa^{\prime}}\right|^{2} \equiv \frac{e^{2} R^{2}}{3} \mathscr{T}_{n j \kappa}^{n^{\prime} j^{\prime} \kappa^{\prime}}, \quad \text { (47a) }
\end{aligned}
$$

with a dimensionless measure of the squared magnitude of the optical dipole vector defined as

$$
\begin{array}{r}
\mathscr{T}_{n j \kappa}^{n^{\prime} j^{\prime} \kappa^{\prime}}=\delta_{\kappa^{\prime}, \kappa} \sum_{\xi= \pm 1} \frac{(2 j+\xi)[2(j+1)+\xi]}{2(2 j+1+\xi)}\left|\frac{\mathscr{R}_{n j}^{n^{\prime} j^{\prime}}}{R}-i \kappa \xi \frac{R_{0}}{2 R} \delta_{n j,-\kappa \xi}^{n^{\prime} j^{\prime}, \kappa \xi}\right|^{2} \delta_{j^{\prime}, j+\xi} \\
+\delta_{\kappa^{\prime},-\kappa} \frac{2 j+1}{4 j(j+1)}\left|\frac{\mathscr{R}_{n j}^{n^{\prime} j^{\prime}}}{R}+i \kappa \frac{R_{0}}{2 R}\left(j \delta_{n j,-\kappa}^{n^{\prime} j^{\prime}, \kappa}+(j+1) \delta_{n j, \kappa}^{n^{\prime} j^{\prime},-\kappa}\right)\right|^{2} \delta_{j^{\prime}, j} .
\end{array}
$$

The nanoparticle-size dependence of $\mathscr{T}_{n j \kappa}^{n^{\prime} j^{\prime} \kappa^{\prime}}$ for the two lowest-energy transitions is plotted in Fig. 4. It exhibits a confinement-induced parity-symmetry breaking that gets most pronounced in the small-size limit where transition amplitudes are dominated by overlap integrals $\delta_{n j, \pm}^{n^{\prime} j^{\prime}, \mp}$ whose magnitude deviates from unity. 

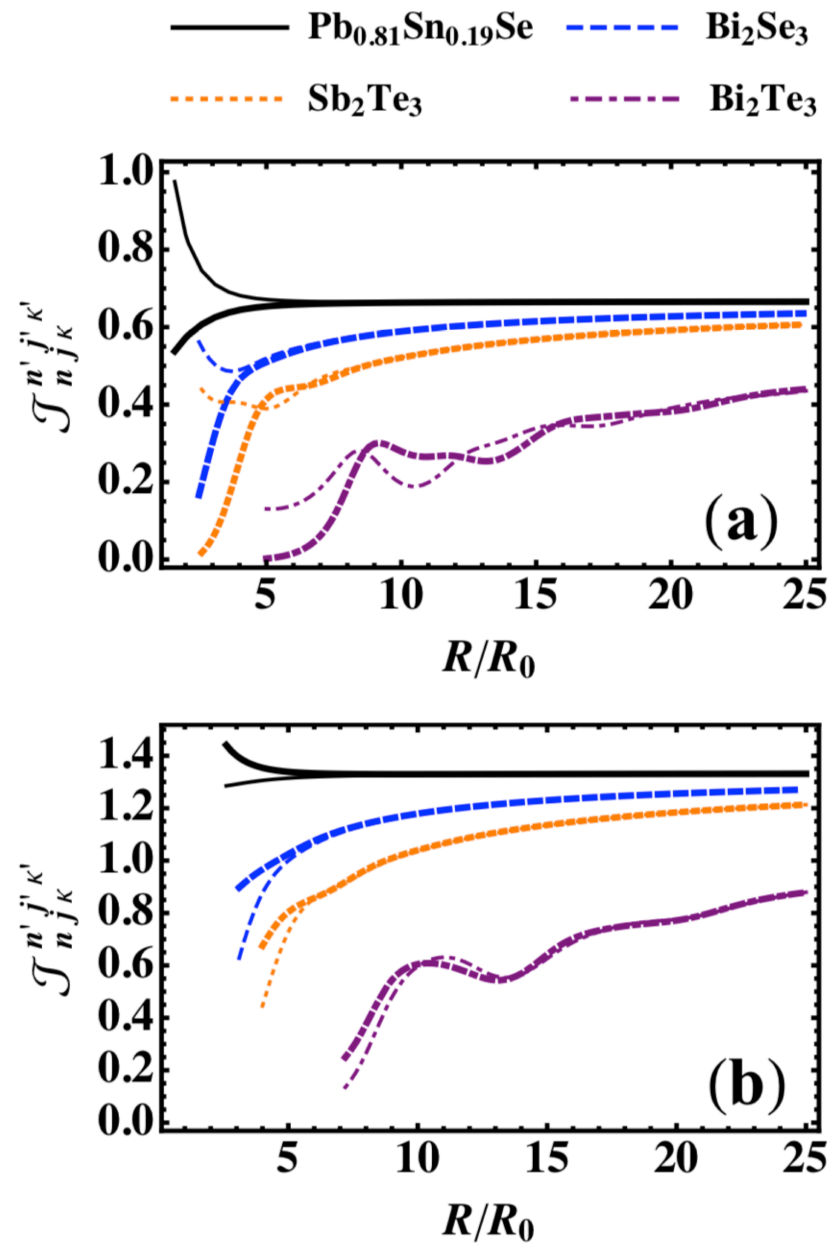

FIG. 4. The modulus squared of the dipole moment for optical transitions in a spherical topological-insulator nanoparticle with radius $R$ is given by $e^{2} R^{2} \mathscr{T}_{n j \kappa}^{n^{\prime} j^{\prime} \kappa^{\prime}}$. Panel (a) plots the nanoparticle-size dependence of the dimensionless dipole magnitude $\mathscr{T}_{n j \kappa}^{n^{\prime} j^{\prime} \kappa^{\prime}}$ for the lowest-energy transitions in an undoped nanoparticle, i.e., for $n=-1, n^{\prime}=1, j=j^{\prime}=1 / 2$, and $\kappa=-\kappa^{\prime}=+/-$ (thick/thin curves). Panel (b) shows $\mathscr{T}_{n j \kappa}^{n^{\prime} j^{\prime} \kappa^{\prime}}$ for the next-highest-energy transitions where $n=-1, n^{\prime}=1$, $j=j^{\prime}-1=1 / 2$, and $\kappa=\kappa^{\prime}=+/-$ (thick/thin curves). Here $R_{0}=2 \gamma /\left|\Delta_{0}\right|$ is the bulk Compton length, and the black solid (blue dashed, orange dotted, purple dot-dashed) curve is obtained for $\Delta_{0} /\left(\gamma k_{\Delta}\right)=-0.039(-1.2,-2.3,-9.5$,$) , which is$ representative of $\mathrm{Pb}_{0.81} \mathrm{Sn}_{0.19} \mathrm{Se}\left(\mathrm{Bi}_{2} \mathrm{Se}_{3}, \mathrm{Sb}_{2} \mathrm{Te}_{3}, \mathrm{Bi}_{2} \mathrm{Te}_{3}\right)$.

\section{CONCLUSIONS AND OUTLOOK}

We have solved analytically the quantum-confinement problem of charge carriers in spherical nanoparticles made from topological-insulator (TI) materials, extending previous results $[25,36]$. The mathematical formalism employed in our study unifies related multi-band descriptions of semiconductor nanocrystals [47, 48] and confined Dirac fermions [32, 35, 36, 56]. Compact transcendental secular equations are obtained for the energies of both ordinary (i.e., deriving from linear combinations of propagating bulk states) and topological (i.e., constituting confined intrinsic surface states) bound-state levels. See Eqs. (28) and (34), respectively. Explicit results for the size dependence of topological-bound-state sub-gap energies calculated using parameters applicable to real materials are shown in Fig. 1. Significant deviations from previously derived $[25,36]$ asymptotic expressions arise already for not-so-small values of $R / R_{0}$, where $R$ and $R_{0}$ are the nanoparticle radius and the bulk-material Compton length, respectively.

A universal form of the envelope-wave-function spinors for TI-nanoparticle bound states in terms of spherical harmonics is presented in Eq. (2). These states are labeled by the half-integer quantum numbers for total angular momentum $(j)$ and its projection onto an arbitrary axis $(m)$, as well as a two-valued quantum number $\kappa= \pm 1$. States with fixed $j, m$ and $\kappa$ are also eigenstates of parity with eigenvalue $(-1)^{j-\frac{\kappa}{2}}$.

We considered optical transitions between singleelectron bound states in a TI nanoparticle via the conventional [65] electric-dipole coupling. Our approach describes both envelope-function- and basis-state-mediated transitions via a compact optical-dipole operator in envelope-function space [see Eqs. (4) and (5)], and general analytical results are obtained for the transition matrix elements [Eqs. (6)]. In addition to the previously discussed [36] transitions between levels where $j^{\prime}-j= \pm 1$, we also identify possible transitions where $j^{\prime}-j=0$. The latter were not identified in Ref. [36]. The possibility to conserve total angular momentum in an optical transition arises because there exist TI-nanoparticle bound states at any given $j$ that have opposite parity. For such a pair of states [see their explicit expressions in Eq. (2)], corresponding spinor entries have orbital angular momentum differing by one unit, as required for accommodating a photon's angular momentum. The multitude of possible transitions between topological bound-state levels in an undoped TI nanoparticle is illustrated in Fig. 3.

The effect of the nanoparticle size on optical transitions is contained in the radial-wave-function overlap integrals given in Eqs. (7) and (8). Their size dependence for the lowest subgap-energy bound state is plotted in Fig. 2. For $R \gg R_{0}$, the envelope-function contribution to the transition matrix element is $\propto R$, reflecting the surfacelocalized character of these states in this limit. However, significant deviations from this expected behavior occur already from $R \sim 10 R_{0}$ for typical materials, and also the basis-state contribution starts to become relevant within this size range. The nanoparticle-radius dependence of transition-dipole magnitudes for the lowestenergy unconventional and conventional types of optical transitions is shown in Fig. 4. Both have similar order of magnitude and exhibit pronounced parity-symmetry breaking in the small- $R$ limit.

Our results provide detailed information about how size quantization affects surface-state-related electronic and optical properties in TI nanoparticles, which has important practical implications. To start with, the exis- 
tence of additional optical transitions beyond those identified earlier in Ref. [36] requires re-evaluation of suggested schemes [36] to employ TI-nanoparticle states in quantum information processing. More generally, our theoretical description can serve as a starting point for developing any type of application that relies on specifics of the electronic energy spectrum and quantum transitions between TI-nanoparticle bound states. For example, some of the currently envisioned uses of TI nanoparticles in optoelectronic $[9,14]$ and thermoelectric [17] devices are contingent on the presence of topologically protected surface states. Recognizing the existence, and strongly materials-dependent magnitude, of a critical radius below which the topological surface states disappear is therefore of crucial importance. The emergent gaplessness of the $\mathrm{Bi}_{2} \mathrm{Te}_{3}$-nanopoarticle spectrum for certain sizes [see Fig. 1(b)] could become a platform for intriguing applications, including the possibility to host unconventional Majorana excitations as was proposed for gapless TI nanoribbons [71].

Our theoretical approach is based on the envelopefunction continuum-model description for the TI band structure [37-39]. The mathematical structure of this formalism necessitates inclusion of fast-decaying wavefunction contributions [those given in Eqs. (24)] representing the effect of remote bands whose influence on observable physical quantities needs to be interpreted with caution [58-60]. Typically, the physically significant wave functions from Eqs. (21) and (32) dominate the system's low-energy properties. However, in confined TI materials with large values of $\left|\Delta_{0}\right| /\left(\gamma k_{\Delta}\right)$, we find that both types of evanescent states [(32) and (24)] present in the sub-gap bound-state wave functions (31) can be relevant on comparable length scales, signaling the impending breakdown of the continuum-model approach. Nevertheless, qualitative trends exhibited in size-dependent physical properties are expected to be accurate even rel- atively close to this extreme limit. Thus our approach serves as a useful tool for modeling the effect of quantum confinement in TI nanoparticles, especially as long as atomistic band-structure calculations [72] can be performed only for very small systems [5, 41]. Results presented here also provide an interesting reference point for understanding size-quantization effects in other TI nanostructures such as cylindrical quantum dots [73] or nanowires with finite [74] and infinite length [75-77].

In future work, the obtained single-particle wave functions for TI-nanoparticle bound states could serve as input to discuss many-body effects due to Coulomb interactions. In particular, exploring the properties of excitons would be of interest in the context of optical properties of TI nanoparticles [24]. The multi-band spinor structure of charge carriers' quantum states should result in a formfactor renormalization of the Coulomb-potential matrix element similar to previously considered situations [78]. Interesting properties associated with two-particle states of Dirac-like carriers [79, 80], e.g., the recently predicted existence of an internal angular momentum of the exciton [81], could be elucidated in greater detail. Early numerical studies [24] can serve as a benchmark for evaluating the accuracy of approximate analytical results.

\section{ACKNOWLEDGMENTS}

The authors benefited from useful discussions with K.I. Imura. The work of M.G.C. was partially supported by a Victoria University of Wellington Summer Research Scholarship. L.G. is the grateful recipient of a Vanier Canada Graduate Scholarship. Research at Perimeter Institute is supported in part by the Government of Canada through the Department of Innovation, Science and Economic Development Canada and by the Province of Ontario through the Ministry of Economic Development, Job Creation and Trade.
[1] A. D. Yoffe, Low-dimensional systems: quantum size effects and electronic properties of semiconductor microcrystallites (zero-dimensional systems) and some quasitwo-dimensional systems, Adv. Phys. 42, 173 (1993).

[2] A. L. Efros and M. Rosen, The electronic structure of semiconductor nanocrystals, Annu. Rev. Mater. Sci. 30, 475 (2000).

[3] S. Cho, D. Kim, P. Syers, N. P. Butch, J. Paglione, and M. S. Fuhrer, Topological insulator quantum dot with tunable barriers, Nano Lett. 12, 469 (2012).

[4] S. V. Kershaw, A. S. Susha, and A. L. Rogach, Narrow bandgap colloidal metal chalcogenide quantum dots: synthetic methods, heterostructures, assemblies, electronic and infrared optical properties, Chem. Soc. Rev. 42, 3033 (2013).

[5] A. Vargas, S. Basak, F. Liu, B. Wang, E. Panaitescu, H. Lin, R. Markiewicz, A. Bansil, and S. Kar, The changing colors of a quantum-confined topological insulator,
ACS Nano 8, 1222 (2014).

[6] G. Z. Jia, W. K. Lou, F. Cheng, X. L. Wang, J. H. Yao, N. Dai, H. Q. Lin, and K. Chang, Excellent photothermal conversion of core/shell CdSe/Bi2Se3 quantum dots, Nano Res. 8, 1443 (2015).

[7] A. Vargas, F. Liu, and S. Kar, Giant enhancement of light emission from nanoscale Bi2Se3, Appl. Phys. Lett. 106, 243107 (2015).

[8] M. S. Claro, I. Levy, A. Gangopadhyay, D. J. Smith, and M. C. Tamargo, Self-assembled bismuth selenide (Bi2Se3) quantum dots grown by molecular beam epitaxy, Sci. Rep. 9, 3370 (2019).

[9] M. S.Rider, M. Sokolikova, S. M. Hanham, M. NavarroCía, P. Haynes, D. Lee, M. Daniele, M. C. Guidi, C. Mattevi, S. Lupi, and V. Giannini, Experimental signature of a topological quantum dot, arXiv:1905.06193 [condmat.mes-hall] (2019).

[10] S. S. Hong, D. Kong, and Y. Cui, Topological insulator 
nanostructures, MRS Bull. 39, 873 (2014).

[11] M. Z. Hasan and C. L. Kane, Colloquium: Topological insulators, Rev. Mod. Phys. 82, 3045 (2010).

[12] M. Z. Hasan and J. E. Moore, Three-dimensional topological insulators, Annu. Rev. Cond. Mat. Phys. 2, 55 (2011).

[13] M. Z. Hasan, S.-Y. Xu, and G. Bian, Topological insulators, topological superconductors and Weyl fermion semimetals: discoveries, perspectives and outlooks, Phys. Scr. T164, 014001 (2015).

[14] A. Politano, L. Viti, and M. S. Vitiello, Optoelectronic devices, plasmonics, and photonics with topological insulators, APL Mater. 5, 035504 (2017).

[15] J. W. McIver, D. Hsieh, H. Steinberg, P. Jarillo-Herrero, and N. Gedik, Control over topological insulator photocurrents with light polarization, Nat. Nanotech. 7, 96 (2012).

[16] J. Tian, S. Hong, I. Miotkowski, S. Datta, and Y. P. Chen, Observation of current-induced, long-lived persistent spin polarization in a topological insulator: A rechargeable spin battery, Sci. Adv. 3, e1602531 (2017).

[17] N. Xu, Y. Xu, and J. Zhu, Topological insulators for thermoelectrics, npj Quantum Mater. 2, 51 (2017).

[18] B. Zhou, H.-Z. Lu, R.-L. Chu, S.-Q. Shen, and Q. Niu, Finite size effects on helical edge states in a quantum spin-Hall system, Phys. Rev. Lett. 101, 246807 (2008).

[19] J. Linder, T. Yokoyama, and A. Sudbø, Anomalous finite size effects on surface states in the topological insulator $\mathrm{Bi}_{2} \mathrm{Se}_{3}$, Phys. Rev. B 80, 205401 (2009).

[20] C.-X. Liu, H. Zhang, B. Yan, X.-L. Qi, T. Frauenheim, X. Dai, Z. Fang, and S.-C. Zhang, Oscillatory crossover from two-dimensional to three-dimensional topological insulators, Phys. Rev. B 81, 041307 (2010).

[21] H.-Z. Lu, W.-Y. Shan, W. Yao, Q. Niu, and S.-Q. Shen, Massive Dirac fermions and spin physics in an ultrathin film of topological insulator, Phys. Rev. B 81, 115407 (2010).

[22] M. Kotulla and U. Zülicke, Manipulating topologicalinsulator properties using quantum confinement, New J. Phys. 19, 073025 (2017).

[23] L. Gioia, U. Zülicke, M. Governale, and R. Winkler, Dirac electrons in quantum rings, Phys. Rev. B 97, 205421 (2018).

[24] N. Malkova and G. W. Bryant, Negative-band-gap quantum dots: Gap collapse, intrinsic surface states, excitonic response, and excitonic insulator phase, Phys. Rev. B 82, 155314 (2010).

[25] K.-I. Imura, Y. Yoshimura, Y. Takane, and T. Fukui, Spherical topological insulator, Phys. Rev. B 86, 235119 (2012).

[26] Y. Takane and K.-I. Imura, Unified description of Dirac electrons on a curved surface of topological insulators, J. Phys. Soc. Jpn. 82, 074712 (2013).

[27] G. Siroki, D. K. K. Lee, P. D. Haynes, and V. Giannini, Single-electron induced surface plasmons on a topological nanoparticle, Nat. Commun. 7, 12375 (2016).

[28] G. Siroki, P. D. Haynes, D. K. K. Lee, and V. Giannini, Protection of surface states in topological nanoparticles, Phys. Rev. Materials 1, 024201 (2017).

[29] H.-G. Zirnstein and B. Rosenow, Time-reversalsymmetric topological magnetoelectric effect in threedimensional topological insulators, Phys. Rev. B 96, 201112 (2017).

[30] T. Neupert, S. Rachel, R. Thomale, and M. Greiter, In- teracting surface states of three-dimensional topological insulators, Phys. Rev. Lett. 115, 017001 (2015).

[31] A. C. Durst, Disorder-induced density of states on the surface of a spherical topological insulator, Phys. Rev. B 93, 245424 (2016).

[32] A. A. Abrikosov, Fermion states on the sphere $S^{2}$, Int. J. Mod. Phys. A 17, 885 (2002), see arXiv:hep-th/0212134 for an extended version that also contains a useful collection of relevant references.

[33] D.-H. Lee, Surface states of topological insulators: The Dirac fermion in curved two-dimensional spaces, Phys. Rev. Lett. 103, 196804 (2009).

[34] V. Parente, P. Lucignano, P. Vitale, A. Tagliacozzo, and F. Guinea, Spin connection and boundary states in a topological insulator, Phys. Rev. B 83, 075424 (2011).

[35] R. Layeghnejad, M. Zare, and R. Moazzemi, Dirac particle in a spherical scalar potential well, Phys. Rev. D 84, 125026 (2011).

[36] H. P. Paudel and M. N. Leuenberger, Three-dimensional topological insulator quantum dot for optically controlled quantum memory and quantum computing, Phys. Rev. B 88, 085316 (2013).

[37] H. Zhang, C.-X. Liu, X.-L. Qi, X. Dai, Z. Fang, and S.-C. Zhang, Topological insulators in $\mathrm{Bi}_{2} \mathrm{Se}_{3}, \mathrm{Bi}_{2} \mathrm{Te}_{3}$, and $\mathrm{Sb}_{2} \mathrm{Te}_{3}$ with a single Dirac cone on the surface, Nat. Phys. 82, 438 (2009).

[38] C.-X. Liu, X.-L. Qi, H. Zhang, X. Dai, Z. Fang, and S.C. Zhang, Model hamiltonian for topological insulators, Phys. Rev. B 82, 045122 (2010).

[39] M. R. Brems, J. Paaske, A. M. Lunde, and M. Willatzen, Symmetry analysis of strain, electric and magnetic fields in the $\mathrm{Bi}_{2} \mathrm{Se}_{3}$-class of topological insulators, New J. Phys. 20, 053041 (2018).

[40] The $\tau_{j}\left(\sigma_{j}\right)$ for $j=x, y, z$ are standard Pauli matrices for the pseudo-spin-1/2 (real-spin-1/2) degree of freedom, and $\tau_{0}\left(\sigma_{0}\right)$ is the identity operator in that subspace. The symbol $\otimes$ indicates the direct product of operators from pseudo-spin and real-spin spaces. The combinations $\sigma_{ \pm} \equiv\left(\sigma_{x} \pm i \sigma_{y}\right) / 2$ are ladder operators for real spin, and $\boldsymbol{\sigma}$ denotes the vector $\left(\sigma_{x}, \sigma_{y}, \sigma_{z}\right)$.

[41] I. A. Nechaev and E. E. Krasovskii, Relativistic k · p Hamiltonians for centrosymmetric topological insulators from ab initio wave functions, Phys. Rev. B 94, 201410 (2016).

[42] E. G. Novik, A. Pfeuffer-Jeschke, T. Jungwirth, V. Latussek, C. R. Becker, G. Landwehr, H. Buhmann, and L. W. Molenkamp, Band structure of semimagnetic $\mathrm{Hg}_{1-y} \mathrm{Mn}_{y}$ Te quantum wells, Phys. Rev. B 72, 035321 (2005).

[43] P. C. Klipstein, Structure of the quantum spin Hall states in $\mathrm{HgTe} / \mathrm{CdTe}$ and InAs/GaSb/AlSb quantum wells, Phys. Rev. B 91, 035310 (2015).

[44] B. A. Assaf, T. Phuphachong, V. V. Volobuev, G. Bauer, G. Springholz, L.-A. de Vaulchier, and Y. Guldner, Magnetooptical determination of a topological index, npj Quant. Mater. 2, 26 (2017).

[45] B. A. Assaf, T. Phuphachong, V. V. Volobuev, A. Inhofer, G. Bauer, G. Springholz, L. A. de Vaulchier, and Y. Guldner, Massive and massless Dirac fermions in $\mathrm{Pb}_{1-x} \mathrm{Sn}_{x}$ Te topological crystalline insulator probed by magneto-optical absorption, Sci. Rep. 6, 20323 (2016).

[46] J. J. Sakurai and J. Napolitano, Modern Quantum Mechanics, 2nd ed. (Addison-Wesley, San Francisco, 2011).

[47] V. I. Sheka and D. I. Sheka, Local states in a semicon- 
ductor with a narrow forbidden band, Zh. Eksp. Teor. Fiz. 51, 1445 (1966), [Sov. Phys. JETP 24, 975 (1967)].

[48] A. L. Efros and M. Rosen, Quantum size level structure of narrow-gap semiconductor nanocrystals: Effect of band coupling, Phys. Rev. B 58, 7120 (1998).

[49] B. Thaller, The Dirac Equation (Springer, Berlin, 1992).

[50] Within a conventional nomenclature [65], the term proportional to the position vector $\mathbf{r}$ (the TI-material Compton length $R_{0}$ ) in Eq. (4) is associated with intra-band (inter-band) transitions. This jargon is somewhat misleading in the case of narrow-gap (e.g., TI) materials [36]. More precisely, the first (second) term on the r.h.s. of Eq. (4) pertains to dipole transitions mediated by the envelope part (the Bloch-function basis) of the confinedelectron states.

[51] W. Greiner, Quantum Mechanics Special Chapters (Springer, Berlin, 1998).

[52] The degree of freedom associated with the quantum number $\sigma$ is the projection of a real spin- $1 / 2$ angular momentum, but this is not the actual electron spin [39].

[53] D. A. Varshalovich, A. N. Moskalev, and V. K. Khersonskii, Quantum Theory of Angular Momentum (World Scientific, Singapore, 1988) Section 7.2.

[54] R. Szmytkowski, Recurrence and differential relations for spherical spinors, J. Math. Chem. 42, 397 (2007).

[55] M. Abramowitz and I. A. Stegun, Handbook of Mathematical Functions (Dover, New York, 1964).

[56] P. Alberto, C. Fiolhais, and V. M. S. Gil, Relativistic particle in a box, Eur. J. Phys. 17, 19 (1996).

[57] B. A. Bernevig, T. L. Hughes, and S. Zhang, Quantum spin Hall effect and topological phase transition in $\mathrm{HgTe}$ quantum wells, Science 314, 1757 (2006).

[58] S. R. White and L. J. Sham, Electronic properties of flatband semiconductor heterostructures, Phys. Rev. Lett. 47, 879 (1981).

[59] M. F. H. Schuurmans and G. W. 't Hooft, Simple calculations of confinement states in a quantum well, Phys. Rev. B 31, 8041 (1985).

[60] P. C. Klipstein, A k · p treatment of edge states in narrow 2D topological insulators, with standard boundary conditions for the wave function and its derivative, J. Phys.: Condens. Matter 30, 275302 (2018).

[61] We find the expressions for $\tilde{k}$ and $\tilde{q}$ by solving for these in the relations $\tilde{E}_{+}^{(\mathrm{D})}(k)=\tilde{E}$ and $\tilde{E}_{+}^{(\mathrm{B})}(q)=\tilde{E}$, respectively. Then $\gamma_{k}$ and $\bar{\gamma}_{q}$ are obtained by inserting the expressions found for $\tilde{k}$ and $\tilde{q}$ into Eqs. (23) and (26), respectively.

[62] B. A. Volkov and O. A. Pankratov, Two-dimensional massless electrons in an inverted contact, Pis'ma Zh. Eksp. Teor. Fiz. 42, 145 (1985), [JETP Lett. 42, 178 (1985)].

[63] A different type of intrinsic semiconductor-nanoparticle sub-gap state discussed in Ref. [82] has its origin in a sign change of effective masses [83] instead of a band inversion.

[64] Using identity 10.2.2 from Ref. [55], it can be shown that the spinors in Eqs. (32) are obtained from those in (21) by making the replacements $k=i \bar{k}$ and $\gamma_{k}=i \bar{\gamma}_{\bar{k}}$.

[65] H. Haug and S. W. Koch, Quantum Theory of the Optical and Electronic Properties of Semiconductors, 4th ed. (World Scientific, Singapore, 2004).
[66] I. Pelant and J. Valenta, Luminescence Spectroscopy of Semiconductors (Oxford University Press, Oxford, UK, 2012).

[67] A. Gustafsson, M.-E. Pistol, L. Montelius, and L. Samuelson, Local probe techniques for luminescence studies of low-dimensional semiconductor structures, J. Appl. Phys. 84, 1715 (1998).

[68] V. M. Fomin, V. N. Gladilin, J. T. Devreese, E. P. Pokatilov, S. N. Balaban, and S. N. Klimin, Photoluminescence of spherical quantum dots, Phys. Rev. B 57, 2415 (1998).

[69] In our notation, initial (final) states are labelled by quantum numbers $n, j, m, \kappa\left(n^{\prime}, j^{\prime}, m^{\prime}, \kappa^{\prime}\right)$. Equation (38) is a generalization of Eq. (5.34) from Ref. [65] and, as the latter, relies on the assumption that the envelope-function part of electronic wave functions varies on much larger length scales than the basis functions.

[70] A. L. Efros and A. L. Efros, Interband absorption of light in a semiconductor sphere, Fiz. Tekh. Poluprovodn. 16, 1209 (1982), [Sov. Phys. Semicond. 16, 772 (1982)].

[71] J. Manousakis, A. Altland, D. Bagrets, R. Egger, and Y. Ando, Majorana qubits in a topological insulator nanoribbon architecture, Phys. Rev. B 95, 165424 (2017).

[72] A. Bansil, H. Lin, and T. Das, Colloquium: Topological band theory, Rev. Mod. Phys. 88, 021004 (2016).

[73] T. M. Herath, P. Hewageegana, and V. Apalkov, A quantum dot in topological insulator nanofilm, J. Phys.: Condens. Matter 26, 115302 (2014).

[74] A. Kundu, A. Zazunov, A. L. Yeyati, T. Martin, and R. Egger, Energy spectrum and broken spin-surface locking in topological insulator quantum dots, Phys. Rev. B 83, 125429 (2011).

[75] K.-I. Imura, Y. Takane, and A. Tanaka, Spin Berry phase in anisotropic topological insulators, Phys. Rev. B 84, 195406 (2011).

[76] P. Iorio, C. A. Perroni, and V. Cataudella, Quantum interference effects in $\mathrm{Bi}_{2} \mathrm{Se}_{3}$ topological insulator nanowires with variable cross-section lengths, Eur. Phys. J. B 89, 97 (2016).

[77] N. Virk, G. Autès, and O. V. Yazyev, Electronic properties of one-dimensional nanostructures of the $\mathrm{Bi}_{2} \mathrm{Se}_{3}$ topological insulator, Phys. Rev. B 97, 165411 (2018).

[78] T. Kernreiter, M. Governale, R. Winkler, and U. Zülicke, Suppression of Coulomb exchange energy in quasi-twodimensional hole systems, Phys. Rev. B 88, 125309 (2013).

[79] J. Sabio, F. Sols, and F. Guinea, Two-body problem in graphene, Phys. Rev. B 81, 045428 (2010).

[80] O. L. Berman, R. Y. Kezerashvili, and K. Ziegler, Coupling of two Dirac particles, Phys. Rev. A 87, 042513 (2013).

[81] M. Trushin, M. O. Goerbig, and W. Belzig, Model prediction of self-rotating excitons in two-dimensional transition-metal dichalcogenides, Phys. Rev. Lett. 120, 187401 (2018).

[82] P. C. Sercel, A. L. Efros, and M. Rosen, Intrinsic gap states in semiconductor nanocrystals, Phys. Rev. Lett. 83, 2394 (1999).

[83] Y. R. Lin-Liu and L. J. Sham, Interface states and subbands in HgTe-CdTe heterostructures, Phys. Rev. B 32, 5561 (1985). 